\title{
Laplace Decomposition Method for Solving Fractional Black- Scholes European Option Pricing Equation
}

\author{
Abiodun Ezekiel Owoyemi ${ }^{1}$, Ira Sumiati ${ }^{2, *}$, Endang Rusyaman ${ }^{3}$, Sukono $^{4}$ \\ ${ }^{I}$ Department of General Studies, Federal, College of Agricultural Produce Technology, Nigeria. \\ ${ }^{2}$ Master Program of Mathematics, Universitas Padjadjaran, Bandung, Indonesia \\ ${ }^{3,4}$ Department of Mathematics, Universitas Padjadjaran, Bandung, Indonesia \\ *Corresponding author email: irasumiati@gmail.com
}

\begin{abstract}
Fractional calculus is related to derivatives and integrals with the order is not an integer. Fractional BlackScholes partial differential equation to determine the price of European-type call options is an application of fractional calculus in the economic and financial fields. Laplace decomposition method is one of the reliable and effective numerical methods for solving fractional differential equations. Thus, this paper aims to apply the Laplace decomposition method for solving the fractional Black-Scholes equation, where the fractional derivative used is the Caputo sense. Two numerical illustrations are presented in this paper. The results show that the Laplace decomposition method is an efficient, easy and very useful method for finding solutions of fractional Black-Scholes partial differential equations and boundary conditions for European option pricing problems.
\end{abstract}

Keywords: Laplace transform, Adomian decomposition method, fractional Black-Scholes equation, Caputo sense, option price.

\section{Introduction}

Fractional calculus is a field of calculus relating to derivatives and integral with the fractional order. In calculus, the derivative has the order of natural number, denoted by $D_{x}^{n} y(x)$, where $n=1,2,3, \ldots$. The notation can be generalized into derivatives of the fractional-order denoted by $D_{x}^{\alpha} y(x)$, where $\alpha \in \mathbb{R}$. Most people assume that fractional calculus is an abstract field of 
mathematics that has very little use and almost no application. Nowadays, various studies have begun to emerge regarding the application of fractional calculus in various fields, such as physics, engineering, chemistry, biology, environment, economics and finance (Debnath, 2003; Kisela, 2008; Dalir and Bashour, 2010; David et al., 2011; Katsikadelis, 2014; Giusti and Colombaro, 2017; Rusyaman et al., 2017; Rusyaman et al., 2018; Sumiati et al., 2018; Sun et al., 2018).

Fractional Black-Scholes partial differential equation to determine option prices is one application of fractional calculus in economics and finance. The classic Black-Scholes equation was first introduced by Fisher Black and Myron Scholes in 1973. The notation $C=C(S, t)$ is the price of European call options when the price of the asset $S$ and time $t$. Let $\sigma$ is the asset price volatility, $E$ is the exercise price, $T$ is the maturity time, and $r$ is the risk-free interest rate. The Black-Scholes equation and the boundary conditions for determining the call option price of European type is (Wilmott et al., 1995; Gulkac, 2010)

$$
\frac{\partial C}{\partial t}+\frac{1}{2} \sigma^{2} S^{2} \frac{\partial^{2} C}{\partial S^{2}}+r S \frac{\partial C}{\partial S}-r C=0
$$

with $C(0, t)=0, C(S, t) \sim S$ as $S \rightarrow \infty$, and $C(S, T)=\max \{S-E, 0\}$. Equation (1) resembles a diffusion equation with more parameters. Equation (1) can be simplified through conversion as follows

$$
S=E e^{x}, t=T-\frac{2 \tau}{\sigma^{2}}, C(S, t)=E v(x, t),
$$

thus a partial differential equation is obtained

$$
\frac{\partial v}{\partial \tau}=\frac{\partial^{2} v}{\partial x^{2}}+(k-1) \frac{\partial v}{\partial x}-k v
$$

where $k=\frac{2 r}{\sigma^{2}}$ and the initial conditions become $v(x, 0)=\max \left\{e^{x}-1,0\right\}$.

Various numerical methods are used to solve fractional Black-Scholes equations, such as homotopy perturbation method (Kumar et al., 2012; Ouafoudi and Gao, 2018), homotopy analysis method (Kumar et al., 2014), variational iteration method (Ghandehari and Ranjbar, 2014a; Eshaghi et al., 2017), finite difference method (Akrami and Erjaee, 2016), projected differential transformation method (Edeki et al., 2017; Edeki et al., 2019) and Adomian decomposition method (Ghandehari and Ranjbar, 2014b; Yavuz and Ozdemir, 2018).

George Adomian (1980) first introduced the Adomian decomposition method to solve the system of stochastic equation. This decomposition method can be an effective and useful procedure for solving differential or integral equations without linearization, perturbation, or discretization (Adomian, 1988). This method is able to solve natural or fractional-order differential equations, ordinary or partial, with initial or boundary value problems, with constant or variable coefficients, linear or nonlinear, homogeneous or non-homogenous (Ray and Bera, 2005; Tatari et al., 2007; Saeed and Rahman, 2010; Duan et al., 2012; Bougoffa and Rach, 2013; Abushammala, 2014; Al awawdah, 2016; Sumiati et al., 2019a). The combination of the Adomian decomposition method with the Laplace transform is used to solve nonlinear differential equations (Khuri, 2001). The 
Laplace decomposition method is efficiently and reliably used to solve Voltera integro-differential equations (Wazwaz, 2010), Burger differential equations (Naghipour and Manafian, 2015), KunduEckhaus equations (Gonzalez-Gaxiola, 2017), system of fractional partial differential equations (Ahmed et al., 2017), the problem of giving up smoking of fractional order (Haq et al., 2018), and classical (non-fractional order) Black-Scholes partial differential equations (Sumiati et al., 2019b).

Based on the background of the problem and previous studies that have been presented, this paper aims to apply the Laplace decomposition method for solving the fractional Black-Scholes partial differential equation and the boundary conditions for the European option pricing problem.

\section{Fractional Calculus}

This section presents the basic theories and properties which relate and support this research, such as the Laplace transform, Mittag-Leffler function, and fractional derivatives.

Definition 1 (Schiff, 1999). Let $f$ be a real or complex function of a variable $t \geq 0$ and $s$ is a real or complex parameter. The Laplace transform of $f$ is defined

$$
F(s)=L[f(t)]=\int_{0}^{\infty} e^{-s t} f(t) d t=\lim _{b \rightarrow \infty} \int_{0}^{b} e^{-s t} f(t) d t,
$$

where the limit value exists and finite or the unnaturally integral is convergent. The inverse of the Laplace transform is denoted as

$$
L^{-1}[F(s)]=f(t), t \geq 0 .
$$

Based on Definition 1 , for $f(t)=t^{n}$ where $t \geq 0$ and $n$ are non-negative integers, the Laplace transform of $f$ is

$$
L\left[t^{n}\right]=\frac{n !}{s^{n+1}}, s>0 .
$$

If $\alpha \in \mathbb{R}$, then equation (4) can be written

$$
L\left[t^{\alpha}\right]=\frac{\Gamma(\alpha+1)}{s^{\alpha+1}}, s>0 .
$$

where $\Gamma(x)$ is the Gamma function. Additionally, based on Definition 1, Laplace transform from derivatives of order $n$ can be written

$$
L\left[f^{(n)}(t)\right]=s^{n} F(s)-s^{n-1} f(0)-s^{n-2} f^{\prime}(0)-\cdots-f^{(n-1)}(0)=s^{n} F(s)-\sum_{k=0}^{n-1} s^{n-k-1} f^{(k)}(0) .
$$

Definition 2 (Mathai and Haubold, 2017). The basic Mittag-Leffler function is denoted by $E_{\alpha}(z)$, where $\alpha \in \mathbb{R}$ and $z \in \mathbb{C}$, is defined as

$$
E_{\alpha}(z)=\sum_{k=0}^{\infty} \frac{z^{k}}{\Gamma(\alpha k+1)}
$$

Definition 3 (Podlubny, 1999). The Riemann-Liouville fractional derivative of the function $f$ for $t$ 
and order $\alpha>0$ defined as

$$
{ }_{a} D_{t}^{\alpha} f(t)=\frac{1}{\Gamma(n-\alpha)} \frac{d^{n}}{d t^{n}} \int_{a}^{t}(t-u)^{n-\alpha-1} f(u) d u, n-1<\alpha \leq n .
$$

Definition 4 (Podlubny, 1999). The Caputo fractional derivative of the function $f$ for $t$ and order $\alpha>0$ defined as

$$
{ }_{a}^{C} D_{t}^{\alpha} f(t)=\frac{1}{\Gamma(n-\alpha)} \int_{a}^{t}(t-u)^{n-\alpha-1} f^{(n)}(u) d u, n-1<\alpha \leq n .
$$

Definition 5 (Podlubny, 1999). The Laplace transform of the Caputo fractional derivative is defined as

$$
L\left[D^{\alpha} f(t)\right]=s^{\alpha} F(s)-\sum_{k=0}^{n-1} s^{\alpha-k-1} f^{(k)}(0), n-1<\alpha \leq n .
$$

\section{Laplace Decomposition Method}

The Adomian decomposition method assumes that the solution is decomposed into an infinite series, the nonlinear term (if any) is decomposed into Adomian polynomials and an iterative algorithm is built to determine the solution recursively. The numerical scheme of Laplace transform based on the Adomian decomposition method applies the Laplace transform and its inverse to the differential equation (Khuri, 2001).

Given the fractional partial differential equation as follows

$$
D_{t}^{\alpha} u(x, t)+N u(x, t)+R u(x, t)=g(x, t),
$$

and initial condition $u(x, 0)=f(x)$, where $D_{t}^{\alpha} \equiv{ }_{0}^{C} D_{t}^{\alpha}$ is the Caputo fractional derivative operator with $0<\alpha \leq 1, N$ is the nonlinear operator, $R$ is the linear operator, $g$ is the function that shows the non-homogenous of the differential equation and $u$ is the function to be determined. Equation (6) can be rewritten as the subject

$$
D_{t}^{\alpha} u(x, t)=g(x, t)-N u(x, t)-R u(x, t) .
$$

Laplace transform of equation (7) is obtained

$$
u(x, s)=\frac{u(x, 0)}{s}+\frac{1}{s^{\alpha}} L[g(x, t)]-\frac{1}{s^{\alpha}} L[N u(x, t)]-\frac{1}{s^{\alpha}} L[R u(x, t)] .
$$

Then, through the inverse Laplace transform to equation (8) is obtained

$$
u(x, t)=u(x, 0)+L^{-1}\left[\frac{1}{s^{\alpha}} L[g(x, t)]\right]-L^{-1}\left[\frac{1}{s^{\alpha}} L[N u(x, t)]\right]-L^{-1}\left[\frac{1}{s^{\alpha}} L[R u(x, t)]\right] .
$$

The Adomian decomposition method assumes that the function $u$ can be decomposed into an infinite series 


$$
u=\sum_{n=0}^{\infty} u_{n}
$$

where $u_{n}$ can be determined recursively. This method also assumes the nonlinear operator $\mathrm{Nu}$ can be decomposed into the infinite polynomial series

$$
N u=\sum_{n=0}^{\infty} A_{n}
$$

where $A_{n}=A_{n}\left(u_{0}, u_{1}, u_{2}, \ldots, u_{n}\right)$ are Adomian polynomials are defined

$$
A_{n}\left(u_{0}, u_{1}, u_{2}, \ldots, u_{n}\right)=\frac{1}{n !} \frac{d^{n}}{d \lambda^{n}}\left[N\left(\sum_{k=0}^{n} \lambda^{k} u_{k}\right)\right]_{\lambda=0}, n=0,1,2, \ldots
$$

where $\lambda$ is a parameter. The Adomian polynomial $A_{n}$ can be described as follows

$$
\begin{aligned}
& A_{0}=\frac{1}{0 !} \frac{d^{0}}{d \lambda^{0}}\left[N\left(\sum_{k=0}^{0} \lambda^{k} u_{k}\right)\right]_{\lambda=0}=N\left(u_{0}\right), \\
& A_{1}=\frac{1}{1 !} \frac{d^{1}}{d \lambda^{1}}\left[N\left(\sum_{k=0}^{1} \lambda^{k} u_{k}\right)\right]_{\lambda=0}=u_{1} N^{\prime}\left(u_{0}\right), \\
& A_{2}=\frac{1}{2 !} \frac{d^{2}}{d \lambda^{2}}\left[N\left(\sum_{k=0}^{2} \lambda^{k} u_{k}\right)\right]_{\lambda=0}=\frac{u_{1}^{2}}{2 !} N^{\prime \prime}\left(u_{0}\right)+u_{2} N^{\prime}\left(u_{0}\right),
\end{aligned}
$$

Substitution of initial conditions, equations (10) and (11) to equation (9), yields

$$
\sum_{n=0}^{\infty} u_{n}=f(x)+L^{-1}\left[\frac{1}{s^{\alpha}} L[g(x, t)]\right]-L^{-1}\left[\frac{1}{s^{\alpha}} L\left[\sum_{n=0}^{\infty} A_{n}\right]\right]-L^{-1}\left[\frac{1}{s^{\alpha}} L\left[R \sum_{n=0}^{\infty} u_{n}\right]\right] .
$$

If both sides of equation (12) are described, then successively is obtained

$$
\begin{aligned}
& u_{0}=f(x)+L^{-1}\left[\frac{1}{s^{\alpha}} L[g(x, t)]\right], \\
& u_{1}=-L^{-1}\left[\frac{1}{s^{\alpha}} L\left[A_{0}\right]\right]-L^{-1}\left[\frac{1}{s^{\alpha}} L\left[R u_{0}\right]\right], \\
& u_{2}=-L^{-1}\left[\frac{1}{s^{\alpha}} L\left[A_{1}\right]\right]-L^{-1}\left[\frac{1}{s^{\alpha}} L\left[R u_{1}\right]\right],
\end{aligned}
$$

thus obtained the recursive relation of the solution of the fractional partial differential equation (6) is as follows

$$
u_{0}=f(x)+L^{-1}\left[\frac{1}{s^{\alpha}} L[g(x, t)]\right]
$$




$$
u_{n+1}=-L^{-1}\left[\frac{1}{s^{\alpha}} L\left[A_{n}\right]\right]-L^{-1}\left[\frac{1}{s^{\alpha}} L\left[R u_{n}\right]\right], n=0,1,2, \ldots
$$

Therefore, the approximate solution of equation (13) is

$$
u \approx \sum_{n=0}^{k} u_{n}, \text { where } \lim _{k \rightarrow \infty} \sum_{n=0}^{k} u_{n}=u
$$

\section{Numerical Illustration}

Two numerical illustrations of the application of the Laplace decomposition method to solve fractional Black-Scholes partial differential equations are presented in this section.

Illustration 1. Based on equation (3), the fractional Black-Scholes partial differential equation is given as follows

$$
\frac{\partial^{\alpha} v}{\partial t^{\alpha}}=\frac{\partial^{2} v}{\partial x^{2}}+(k-1) \frac{\partial v}{\partial x}-k v
$$

with the initial condition $v(x, 0)=\max \left\{e^{x}-1,0\right\}$.

The solution of the fractional Black-Scholes equation (15) using the Laplace decomposition method is as follows

$$
\begin{aligned}
v_{0} & =\max \left\{e^{x}-1,0\right\} \\
v_{n+1} & =L^{-1}\left[\frac{1}{s^{\alpha}} L\left[\frac{\partial^{2} v_{n}}{\partial x^{2}}+(k-1) \frac{\partial v_{n}}{\partial x}-k v_{n}\right]\right], n=0,1,2, \ldots
\end{aligned}
$$

If the recursive solution is described, then it is obtained

$$
\begin{aligned}
v_{1} & =L^{-1}\left[\frac{1}{s^{\alpha}} L\left[\frac{\partial^{2} v_{0}}{\partial x^{2}}+(k-1) \frac{\partial v_{0}}{\partial x}-k v_{0}\right]\right] \\
& =L^{-1}\left[\frac{1}{s^{\alpha}} L\left[k \max \left\{e^{x}, 0\right\}-k \max \left\{e^{x}-1,0\right\}\right]\right] \\
& =L^{-1}\left[\frac{k \max \left\{e^{x}, 0\right\}-k \max \left\{e^{x}-1,0\right\}}{s^{\alpha+1}}\right] \\
& =\frac{t^{\alpha}}{\Gamma(\alpha+1)}\left(k \max \left\{e^{x}, 0\right\}-k \max \left\{e^{x}-1,0\right\}\right),
\end{aligned}
$$

because $\frac{\partial v_{1}}{\partial x}=\frac{t^{\alpha}}{\Gamma(\alpha+1)}\left(k \max \left\{e^{x}, 0\right\}-k \max \left\{e^{x}, 0\right\}\right)=0$, so that

$$
\begin{aligned}
v_{2} & =L^{-1}\left[\frac{1}{s^{\alpha}} L\left[\frac{\partial^{2} v_{1}}{\partial x^{2}}+(k-1) \frac{\partial v_{1}}{\partial x}-k v_{1}\right]\right] \\
& =L^{-1}\left[\frac{1}{s^{\alpha}} L\left[\frac{t^{\alpha}}{\Gamma(\alpha+1)}\left(-k^{2} \max \left\{e^{x}, 0\right\}+k^{2} \max \left\{e^{x}-1,0\right\}\right)\right]\right]
\end{aligned}
$$




$$
\begin{aligned}
& =L^{-1}\left[\frac{-k^{2} \max \left\{e^{x}, 0\right\}+k^{2} \max \left\{e^{x}-1,0\right\}}{s^{2 \alpha+1}}\right] \\
& =\frac{t^{2 \alpha}}{\Gamma(2 \alpha+1)}\left(-k^{2} \max \left\{e^{x}, 0\right\}+k^{2} \max \left\{e^{x}-1,0\right\}\right), \\
v_{3} & =L^{-1}\left[\frac{1}{s^{\alpha}} L\left[\frac{\partial^{2} v_{2}}{\partial x^{2}}+(k-1) \frac{\partial v_{2}}{\partial x}-k v_{2}\right]\right] \\
& =L^{-1}\left[\frac{1}{s^{\alpha}} L\left[\frac{t^{2 \alpha}}{\Gamma(2 \alpha+1)}\left(k^{3} \max \left\{e^{x}, 0\right\}-k^{3} \max \left\{e^{x}-1,0\right\}\right)\right]\right] \\
& =L^{-1}\left[\frac{k^{3} \max \left\{e^{x}, 0\right\}-k^{3} \max \left\{e^{x}-1,0\right\}}{s^{2 \alpha+1}}\right] \\
& =\frac{t^{3 \alpha}}{\Gamma(3 \alpha+1)}\left(k^{3} \max \left\{e^{x}, 0\right\}-k^{3} \max \left\{e^{x}-1,0\right\}\right),
\end{aligned}
$$

$\vdots$

Therefore, the solution of the fractional Black-Scholes equation (15) can be formed into an infinite series which converges as follows

$$
v(x, t)=\sum_{n=0}^{\infty} v_{n}(x, t)=\max \left\{e^{x}-1,0\right\} E_{\alpha}\left(-k t^{\alpha}\right)+\max \left\{e^{x}, 0\right\}\left(1-E_{\alpha}\left(-k t^{\alpha}\right)\right)
$$

For $\alpha=1$, then obtained

$$
v(x, t)=\max \left\{e^{x}-1,0\right\} e^{-k t}+\max \left\{e^{x}, 0\right\}\left(1-e^{-k t}\right),
$$

the above solution is equivalent to the exact solution of the Black-Scholes partial differential equation (Ghandehari and Ranjbar, 2014b; Yavuz and Ozdemir, 2018; Sumiati et al., 2019c).

Figure 1 is a graph of the solution of the fractional Black-Scholes equation (17), respectively for $\alpha$ equal to $0.2,0.75,0.8$, and 1.0 , where $k$ equal to 0.5 . 


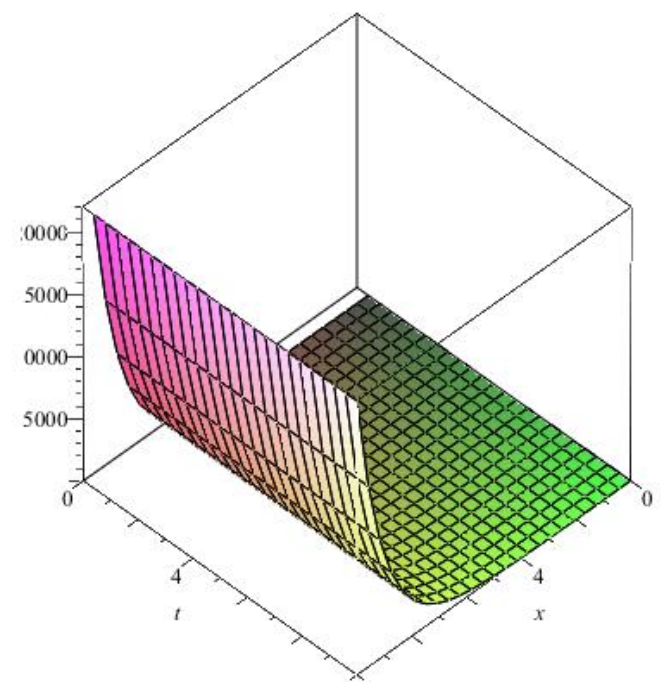

(a)

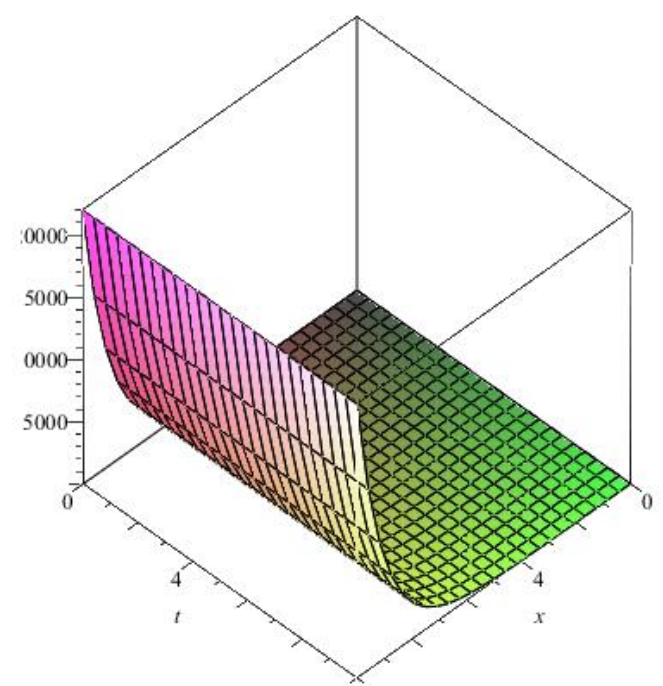

(c)

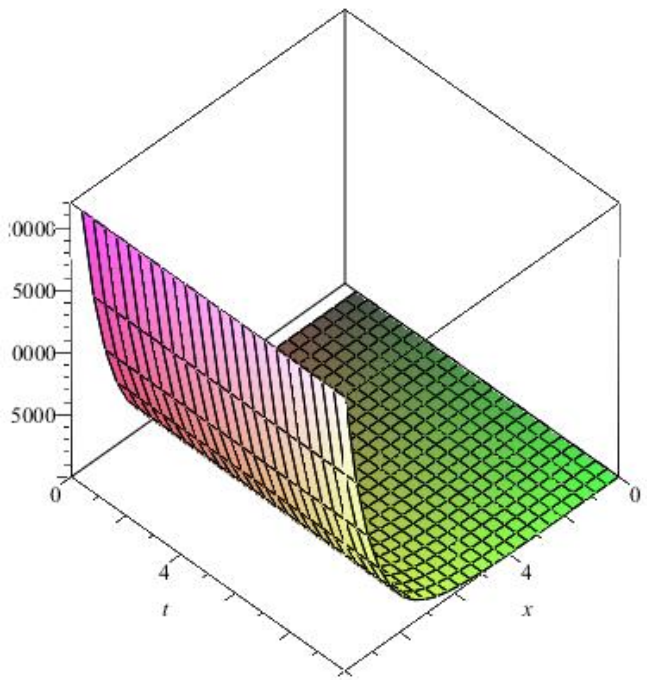

(b)

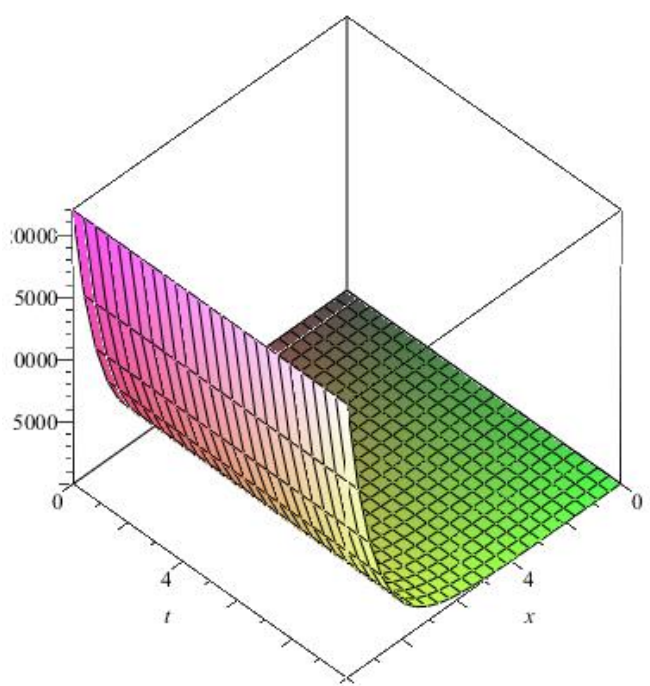

(d)

Figure 1. Graph of the solution function (17) with different $\alpha$ values: (a) $\alpha=0.2$; (b) $\alpha=0.75$; (c) $\alpha=0.8$; (d) $\alpha=1$

Illustration 2. Given the generalization of fractional Black-Scholes partial differential equations as follows (Cen and Le, 2011; Kumar et al., 2012; Yavuz and Ozdemir, 2018)

$$
\frac{\partial^{\alpha} v}{\partial t^{\alpha}}+0.08(2+\sin x)^{2} x^{2} \frac{\partial^{2} v}{\partial x^{2}}+0.06 x \frac{\partial v}{\partial x}-0.06 v=0,
$$

with the initial condition $v(x, 0)=\max \left\{x-25 e^{-0.06}, 0\right\}$.

The solution of the fractional Black-Scholes equation (18) using the Laplace decomposition 
method is as follows

$$
\begin{aligned}
v_{0} & =\max \left\{x-25 e^{-0.06}, 0\right\} \\
v_{n+1} & =L^{-1}\left[\frac{1}{s^{\alpha}} L\left[-0.08(2+\sin x)^{2} x^{2} \frac{\partial^{2} v_{n}}{\partial x^{2}}-0.06 x \frac{\partial v_{n}}{\partial x}+0.06 v_{n}\right]\right], n=0,1,2, \ldots
\end{aligned}
$$

If the recursive solution is described, then it is obtained

$$
\begin{aligned}
v_{1} & =L^{-1}\left[\frac{1}{s^{\alpha}} L\left[-0.08(2+\sin x)^{2} x^{2} \frac{\partial^{2} v_{0}}{\partial x^{2}}-0.06 x \frac{\partial v_{0}}{\partial x}+0.06 v_{n}\right]\right] \\
& =L^{-1}\left[\frac{1}{s^{\alpha}} L\left[-0.06 x+0.06 \max \left\{x-25 e^{-0.06}, 0\right\}\right]\right] \\
& =L^{-1}\left[\frac{-0.06 x+0.06 \max \left\{x-25 e^{-0.06}, 0\right\}}{s^{\alpha+1}}\right] \\
& =\frac{t^{\alpha}}{\Gamma(\alpha+1)}\left(-0.06 x+0.06 \max \left\{x-25 e^{-0.06}, 0\right\}\right),
\end{aligned}
$$

because $\frac{\partial v_{1}}{\partial x}=\frac{t^{\alpha}}{\Gamma(\alpha+1)}(-0.06+0.06)=0$, so that

$$
\begin{aligned}
v_{2} & =L^{-1}\left[\frac{1}{s^{\alpha}} L\left[-0.08(2+\sin x)^{2} x^{2} \frac{\partial^{2} v_{1}}{\partial x^{2}}-0.06 x \frac{\partial v_{1}}{\partial x}+0.06 v_{1}\right]\right] \\
& =L^{-1}\left[\frac{1}{s^{\alpha}} L\left[\frac{t^{\alpha}}{\Gamma(\alpha+1)}\left(-(0.06)^{2} x+(0.06)^{2} \max \left\{x-25 e^{-0.06}, 0\right\}\right)\right]\right] \\
& =L^{-1}\left[\frac{-(0.06)^{2} x+(0.06)^{2} \max \left\{x-25 e^{-0.06}, 0\right\}}{s^{2 \alpha+1}}\right] \\
& =\frac{t^{2 \alpha}}{\Gamma(2 \alpha+1)}\left(-(0.06)^{2} x+(0.06)^{2} \max \left\{x-25 e^{-0.06}, 0\right\}\right), \\
v_{3} & =L^{-1}\left[\frac{1}{s^{\alpha}} L\left[-0.08(2+\sin x)^{2} x^{2} \frac{\partial^{2} v_{2}}{\partial x^{2}}-0.06 x \frac{\partial v_{2}}{\partial x}+0.06 v_{2}\right]\right] \\
& =L^{-1}\left[\frac{1}{s^{\alpha}} L\left[\frac{t^{2 \alpha}}{\Gamma(2 \alpha+1)}\left(-(0.06)^{3} x+(0.06)^{3} \max \left\{x-25 e^{-0.06}, 0\right\}\right)\right]\right. \\
& =L^{-1}\left[\frac{-(0.06)^{3} x+(0.06)^{3} \max \left\{x-25 e^{-0.06}, 0\right\}}{s^{2 \alpha+1}}\right] \\
& =\frac{t^{3 \alpha}}{\Gamma(3 \alpha+1)}\left(-(0.06)^{3} x+(0.06)^{3} \max \left\{x-25 e^{-0.06}, 0\right\}\right),
\end{aligned}
$$

Therefore, the solution of generalizing the fractional Black-Scholes equation (18) is 


$$
v(x, t)=\sum_{n=0}^{\infty} v_{n}(x, t)=\max \left\{x-25 e^{-0.06}, 0\right\} E_{\alpha}\left(0.06 t^{\alpha}\right)+x\left(1-E_{\alpha}\left(0.06 t^{\alpha}\right)\right) .
$$

For $\alpha=1$, then obtained

$$
v(x, t)=\max \left\{x-25 e^{-0.06}, 0\right\} e^{0.06 t}+x\left(1-e^{0.06 t}\right),
$$

the above solution is equivalent to the exact solution of the generalized Black-Scholes partial differential equation (Ghandehari and Ranjbar, 2014b; Yavuz and Ozdemir, 2018; Sumiati et al., 2019c).

Figure 2 is a graph of the solution of the generalized fractional Black-Scholes equation (20), respectively for $\alpha$ equal to $0.2,0.75,0.9$, and 1.0 .

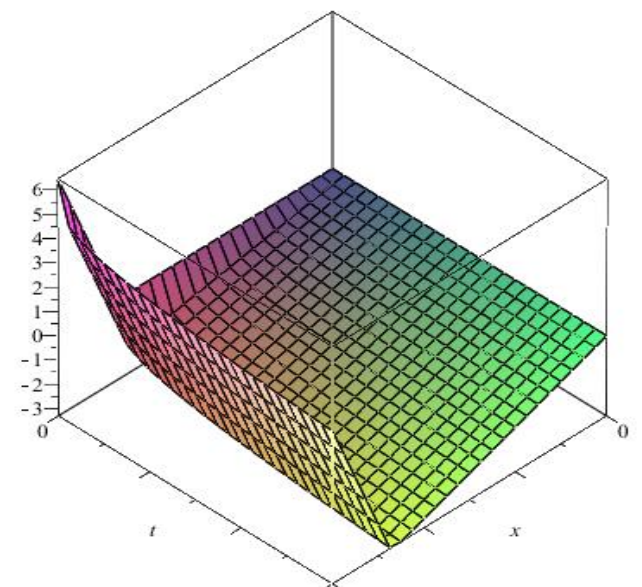

(a)

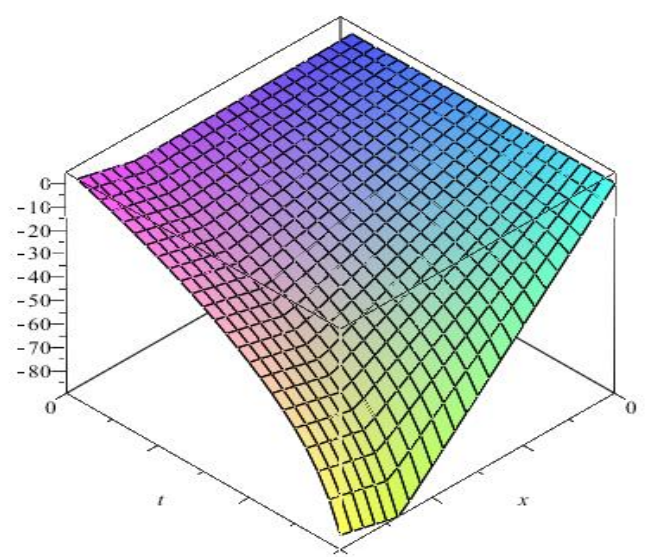

(c)

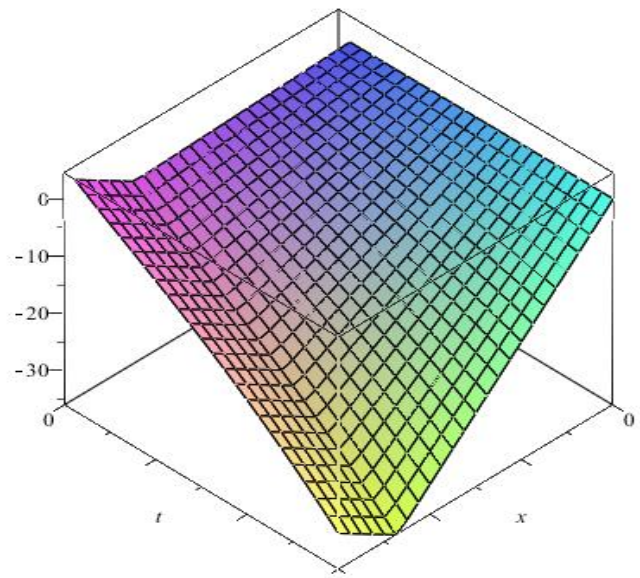

(b)

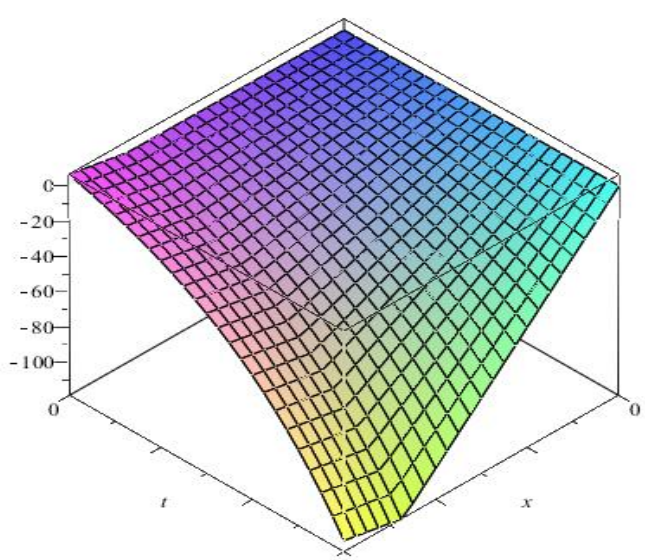

(d)

Figure 2. Graph of the solution function (20) with different $\alpha$ values: (a) $\alpha=0.2$; (b) $\alpha=0.75$; (c) $\alpha=0.9$; (d) $\alpha=1$ 
Based on the two numerical illustrations given, Laplace decomposition method has the advantage that the solution can be expressed as an infinite series that converges quickly to its exact solution. This is supported by the results of the analysis of the solution function and its graph, showing that the fractional-order moves from 0.2 to 1 , then the solution function graph also converges towards the exact solution.

\section{Conclusion}

The Laplace decomposition method is a combination of the Adomian decomposition method and the Laplace integral transform. This method is very reliable and able to solve ordinary or partial differential equations and can be ordered natural or fractional number. The two numerical illustrations are presented in this paper show that the Laplace decomposition method is very effective, useful and easy to use for solving fractional Black-Scholes partial differential equations with boundary conditions for European option pricing problems.

\section{References}

Abushammala, M. B. H. (2014). Iterative Methods for the Numerical Solutions of Boundary Value Problems. A Thesis Presented to the Faculty of the American University of Sharjah College of Arts and Sciences in Partial Fulfilment of the Requirements for the Degree of Master of Science in Mathematics.

Adomian, G. (1980). Stochastic Systems Analysis. Applied Stochastic Processes, 1-17.

Adomian, G. (1988). A Review of the Decomposition Method in Applied Mathematics. Journal of Mathematical Analysis and Applications, 135, 501-544.

Ahmed, H. F., Bahgat, M. S. M., and Zaki, M. (2017). Numerical approaches to system of fractional partial differential equations. Journal of the Egyptian Mathematical Society, 25, 141-150.

Akrami, M. H., and Erjaee, G. H. (2016). Numerical Solution for Fractional Black-Scholes Option Pricing Equation. Global Analysis and Discrete Mathematics, 1(1), 9-14.

Al-awawdah, E. (2016). The Adomian Decomposition Method for Solving Partial Differential Equations. Thesis of Department of Mathematics, Birzeit University, Palestine.

Black, F., and Scholes, M. (1973). The Pricing of Options and Corporate Liabilities. The Journal of Political Economy, 81(3), 637-654.

Bougoffa, L., and Rach, R. C. (2013). Solving nonlocal initial-boundary value problems for linear and 
nonlinear parabolic and hyperbolic partial differential equations by the Adomian decomposition method. Applied Mathematics and Computation, 225, 50-61.

Cen, Z., and Le, A. (2011). A robust and accurate finite difference method for a generalized Black-Scholes equation. Journal of Computational and Applied Mathematics, 235, 3728-3733.

Dalir, M., and Bashour, M. (2010). Applications of Fractional Calculus. Applied Mathematical Sciences, 4(21), 1021-1032.

David, S. A., Linares, J. L., and Pallone, E. M. J. A. (2011). Fractional order calculus: historical apologia basic concepts and some applications. Revista Brasileira de Ensino de Física, 33(4), 4302(1-7).

Debnath, L. (2003). Recent Applications of Fractional Calculus to Science and Engineering. IJMMS, 54, 3413-3442.

Duan, J., Rach, R., Baleanu, D., and Wazwaz, A. (2012). A review of the Adomian decomposition method and its applications to fractional difierential equations. Commun. Frac. Calc., 3(2), 73-99.

Edeki, S. O., Ugbebor, O. O., and Owoloko, E. A. (2017). Analytical Solutions of the Time-fractional Order Black-Scholes Model for Stock Option Valuation on No Dividend Yield Basis. IAENG International Journal of Applied Mathematics, 47(4), 1-12.

Edeki, S. O., Ugbebor, O. O., and Ogundile, O. O. (2019). Analytical Solutions of a Continuous Arithmetic Asian Model for Option Pricing using Projected Differential Transform Method. Engineering Letters, 27(2), 23-34.

Eshaghi S., Ansari, A., Ghaziani, R. K., and Darani, M. A. (2017). Fractional Black-Scholes Model with Regularized Prabhakar Derivative. Publications de l'Institut Mathématique, 102(116), 121-132.

Ghandehari, M. A. M., and Rajbar, M. (2014a). European option pricing of fractional Black-Scholes model with new Lagrange multipliers. Computational Methods for Differential Equations, 2(1), 1-10.

Ghandehari, M. A. M., and Rajbar, M. (2014b). European Option Pricing of Fractional Version of the BlackScholes Model: Approach Via Expansion in Series. International Journal of Nonlinear Science, 17(2), 105-110.

Giusti, A., and Colombaro, I. (2017). Prabhakar-like Fractional Viscoelasticity. Communications in Nonlinear Science and Numerical Simulation. doi: 10.1016/j.cnsns.2017.08.002

González-Gaxiola, O. (2017). The Laplace-Adomian Decomposition Method Applied to the Kundu-Eckhaus Equation. International Journal of Mathematics and Its Applications, 5(1-A), 1-12. 
Gülkaç, V. (2010). The homotopy perturbation method for the Black-Scholes equation. Journal of Statistical Computation and Simulation, 80(12), 1349-1354.

Haq, F., Shah, K., Rahman, G., and Shahzad, M. (2018). Numerical solution of fractional order smoking model via laplace Adomian decomposition method. Alexandria Engineering Journal, 57, 1061-1069.

Katsikadelis, J. T. (2014). Generalized fractional derivatives and their applications to mechanical systems. Arch Appl Mech. doi: 10.1007/s00419-014-0969-0

Khuri, S. A. (2001). A Laplace Decomposition Algorithm Applied to a Class of Nonlinear Differential Equations. Journal of Applied Mathematics, 1(4), 141-155.

Kisela, T. (2008). Fractional Differential Equations and Their Applications. Diploma Thesis of Institute of Mathematics, Faculty of Mechanical Engineering, Brno University of Technology.

Kumar, S., Yildirim, A., Khan, Y., Jafari, H., Sayevand, K., and Wei, L. (2012). Analytical Solution of Fractional Black-Scholes European Option Pricing Equation by Using Laplace Transform. Journal of Fractional Calculus and Application, 2(8), 1-9.

Kumar, S., Kumar, D., and Singh, J. (2014). Numerical computation of fractional Black-Scholes equation arising in financial market. Egyptian Journal of Basic and Applied Sciences, 1, 177-183.

Mathai, A. M., and Haubold, H. J. (2017). An Introduction to Fractional Calculus. New York: Nova Science Publishers.

Naghipour, A., and Manafian, J. (2015). Application of the Laplace Adomian Decomposition and Implicit Methods for Solving Burgers’ Equation. TWMS J. Pure Appl. Math., 6(1), 68-77.

Ouafoudi, M., and Gao, F. (2018). Exact Solution of Fractional Black-Scholes European Option Pricing Equations. Applied Mathematics, 9, 86-100.

Podlubny, I. (1999). Fractional Differential Equations. California: Academis Press.

Ray, S. S., and Bera, R. K. (2005). An approximate solution of a nonlinear fractional differential equation by Adomian decomposition method. Applied Mathematics and Computation, 167, 561-571.

Rusyaman, E., Carnia, E., Parmikanti, K., Sudradjat, S., and Supriatna, A. K. (2017). The Effect of Surface Pressure and Elasticity to the Surface Minimum Energy with Fractional Order. Journal of Engineering and Applied Sciences, 12(19), 4851-4855. 
Rusyaman, E., Chaerani, D., and Parmikanti, K. (2018). Fractional Differential Equation as a Models of Newton Fluids for Stress and Strain Problems. Advances in Social Science, Education and Humanities Research, 218, 34-53.

Saeed, R. K., and Rahman, B. M. (2010). Adomian Decomposition Method for Solving System of Delay Differential Equation. Australian Journal of Basic and Applied Sciences, 4(8), 3613-3621.

Schiff, J. L. (1999). The Laplace Transform: Theory and Applications. New York: Springer.

Sumiati, I., Rusyaman, E., Subartini, B., Sukono, and Budiono, R. (2018). The application of fractional derivatives through Riemannliouville approach to xanthan gum viscoelasticity. International Journal of Engineering \& Technology, 7(4), 2633-2637.

Sumiati, I., Rusyaman, E., Sukono, Subiyanto, and Bon, A. T. (2019a). A Review of Adomian Decomposition Method and Applied to Deferential Equations. Proceedings of the International Conference on Industrial Engineering and Operations Management, Pilsen, Czech Republic, July 23-26, 1328-1338.

Sumiati, I., Rusyaman, E., and Sukono. (2019b). Black-Scholes Equation Solution Using Laplace-Adomian Decomposition Method. IAENG International Journal of Computer Science, 46(4), 707-724.

Sumiati, I., Rusyaman, E., Sukono, and Bon, A. T. (2019c). Sumudu Decomposition Method for BlackScholes Option Pricing Equation. Proceedings of the International Conference on Industrial Engineering and Operations Management, Pilsen, Czech Republic, July 23-26, 2409-2416.

Sun, H., Zhang, Y., Baleanu, D., Chen, W., and Chen, Y. (2018). A new collection of real-world applications of fractional calculus in science and engineering, Communications in Nonlinear Science and Numerical Simulation. doi: 10.1016/j.cnsns.2018.04.019

Tatari, M., Dehgan, M., and Razzaghi, M. (2007). Application of the Adomian decomposition method for the Fokker-Planck equation. Mathematical and Computer Modelling, 45, 639-650.

Wazwaz, A. (2010). The combined Laplace transform-Adomian decomposition method for handling nonlinear Volterra integro-differential equations. Applied Mathematics and Computation, 216, 13041309.

Wilmott, P., Howison, S., and Dewyne, J. (1995). The Mathematics of Financial Derivarives, first edition. Cambrige: Cambrige University Press.

Yavuz, M., and Özdemir, N. (2018). A Quantitative Approach to Fractional Option Pricing Problems with Decomposition Series. Konuralp Journal of Mathematics, 6(1), 102-109. 